This is the final peer-reviewed accepted manuscript of:

Maria De Angelis, Lucia Vannini, Raffaella Di Cagno, Noemi Cavallo, Fabio Minervini, Ruggiero Francavilla, Danilo Ercolini, Marco Gobbetti. Salivary and fecal microbiota and metabolome of celiac children under gluten-free diet, which has been published in final form in International Journal of Food Microbiology Volume 239, 19 December 2016, Pages 125-132

The final published version is available online at: https://doi.org/10.1016/j.ijfoodmicro.2016.07.025

(C) 2016 Elsevier. This manuscript version is made available under the Creative Commons Attribution-NonCommercialNoDerivs (CC BY-NC-ND) 4.0 International License

(http://creativecommons.org/licenses/by-nc-nd/4.0/)

\title{
Salivary and fecal microbiota and metabolome of celiac children under gluten-free diet
}

\author{
Maria De Angelis, Lucia Vannini, Raffaella Di Cagno, Noemi Cavallo, Fabio Minervini, Ruggiero \\ Francavilla, Danilo Ercolini, Marco Gobbetti \\ a Department of Soil, Plant and Food Science, University of Bari Aldo Moro, Via G. Amendola 165/a, 70126 Bari, Italy \\ ${ }^{\mathrm{b}}$ Department of Agricultural and Food Sciences, University of Bologna, Bologna, Italy \\ c Department of Interdisciplinary Medicine, University of Bari Aldo Moro, Bari, Italy \\ d Department of Agricultural Sciences, Division of Microbiology, University of Naples Federico II, Portici, Italy
}

\section{A B S T R A C T}

Celiac disease (CD) is an inflammatory autoimmune disorder resulting from the combination of genetic predis-position and gluten ingestion. A life-long gluten free diet (GFD) is the only therapeutic approach. Dysbiosis, which can precede the CD pathogenesis and/or persist when subjects are on GFD, is reviewed and discussed. Sal-ivary microbiota and metabolome differed between healthy and celiac children treated under GFD (T-CD) for at least two years. The type of GFD (African- vs Italian-style) modified the microbiota and metabolome of Saharawi T-CD children. Different studies showed bacterial dysbiosis at duodenal and/or fecal level of patients with active untreated CD (U-CD) and T-CD compared to healthy subjects. The ratio of protective anti-inflammatory bacteria such as LactobacillusBifidobacterium to potentially harmful Bacteroides-Enterobacteriaceae was the lowest in U-CD and T-CD children. In agreement with dysbiosis, serum, fecal and urinary metabolome from U-CD and T-CD patients showed altered levels of free amino acids and volatile organic compounds. However, consensus across studies defining specific bacteria and metabolites in $\mathrm{U}-\mathrm{CD}$ or T-CD patients is still lacking.

Future research efforts are required to determine the relationships between $\mathrm{CD}$ and oral and intestinal microbiotas to improve the composition of GFD for restoring the gut dysbiosis as a preventative or therapeutic approach for $\mathrm{CD}$.

Keywords:

Celiac disease

Microbiota

Metabolome

Gluten-free diet

\section{Introduction}

Celiac disease (CD) is an autoimmune condition secondary to an immunological response to ingested gluten, in genetically susceptible individuals. $\mathrm{CD}$ is characterized by the presence of a variable combination of gluten-dependent clinical manifestations, CD-specific antibodies (antitransglutaninase IgA, anti-endomisial-IgA and anti-deamidated gliadin-IgA), HLA-DQ2 or HLA-DQ8 haplotypes, and enteropathy. CD is triggered by the ingestion of gliadin and related prolamins (wheat, barley and rye) that are rich in glutamine and proline residues and resist to mammalian protease digestion, creating toxic immunogenic peptides. These peptides have glutamine residues that are preferably susceptible to deamination by tissue transglutaminase (tTG). This modification makes the peptides recognizable by HLA-DQ2 molecule of antigen-presenting cells (DQ2 + or DQ8 + ). In turn, DQ2 + or DQ8 + cells submit gluten peptides to CD4 + T cells that trigger the T-helper-cell type- 1 response, with the consequent, gamma interferon-mediated, development of celiac lesion. The latter is represented by intraepithelial and

\footnotetext{
* Corresponding author.

E-mail address: maria.deangelis@uniba.it (M. De Angelis).
}

lamina propria infiltration of inflammatory cells, crypt hyperplasia, and villous atrophy (Stepniak and Koning, 2006).

CD was thought to affect exclusively Europeans. However, it is distributed worldwide, affecting 0.6 to $1.0 \%$ of the world's population (Fasano and Catassi, 2012; Lionetti et al., 1999; Stepniak and Koning, 2006). Epidemiological studies in Africa, Middle East, Asia, and South America have shown that CD is present but mainly underdiagnosed, with areas of higher prevalence, such as the Saharawi population in North Africa where the highest prevalence of CD (5.6\%) known in the world today is registered (Teresi et al., 2010). CD commonly appears in early childhood, with typical symptoms including chronic diarrhea, abdominal distension, and failure to thrive or atypical symptoms such as short stature, iron deficiency anemia, hypertransaminasemia, oral ulcers or enamel defect. However, symptoms may not develop until later in life, when the disease occurs with fatigue, weight loss, anemia, neurological symptoms and or complications (auto-immune conditions). CD is a life-long disorder and, if untreated, is associated with increased morbidity and mortality (Di Sabatino and Corazza, 2009).

Once the diagnosis is achieved, the therapeutic approach is a strict, life-long gluten free diet (GFD). However, complete exclusion of gluten is difficult due to the ubiquitous nature of this protein and cross-contamination of foods. Selected sourdough biotechnology applied for the 
production of gluten-free leavened products could be useful to eliminate gluten contamination (Di Cagno et al., 2008; Gobbetti et al., 2014). Barley glutamine-specific endoprotease, prolyl-endopeptidase from Sphingomonas capsulata, peptidase form selected sourdough lactic acid bacteria, prolyl-endoprotease from Aspergillus niger showed the capacity to digest gluten under gastrointestinal conditions (Mitea et al., 2008; De Angelis et al., 2010; Caputo et al., 2010). Indeed, alternative therapeutic options could be the oral supplementation of oligopeptidases to hydrolyze gluten immunogenic peptides.

$\mathrm{CD}$ commonly appears in children after the first exposures to gluten, but an increasing number of patients is also experiencing CD onset in late adulthood or adult age, suggesting that additional environmental factors could play a role in CD development (Cenit et al., 2015). An increasing number of evidences strongly indicate that oral and intestinal microbiotas play a central role in human health and disease (Claesson et al., 2012; Ling et al., 2013; Petersen, 2003; Zarco et al., 2012). A condition of dysbiosis is associated with CD patients at the diagnosis but also in remission (e.g., after two years of GFD) (for reviews see Cenit et al., 2015; Francavilla et al., 2014; Marasco et al., 2016; Verdu et al., 2015).

It was suggested that intestinal dysbiosis is strongly correlated with the pathogenesis and progression of several gastro-intestinal diseases such as dyspepsia, diarrhea, Inflammatory bowel disease (IBD), colorectal cancer (CRC), CD, and Irritable bowel syndrome (IBS) (Rautava et al., 2012). Genetic predisposition and environmental factors (e.g., diet, appendectomy, and antibiotic use) could be involved in the intestinal dysbiosis. Subjects at risk of developing CD show an association between altered microbiota and genes, which encode factors for bacterial sensing, immune reaction and metabolism. HLA-DQ2 and -DQ8 genotypes, therefore, select for pathobionts at an early stage, before the clinical onset of $\mathrm{CD}$. Such an altered microbial composition also induces down-regulation of genes, which usually mark the healthy balance (Galipeau et al., 2015; Verdu et al., 2015).

Current data suggest that diet is a major driver of the composition and function of intestinal microbiota and could serve as a means of therapeutic intervention for prevention of diseases (Sonnenburg and Bäckhed, 2016; Voreades et al., 2014; Albenberg and Wu, 2014; Flint et al., 2015; Jeffery and O'Toole, 2013). GFD, per se, may affect the composition of gut microbiota and metabolome (Baranska et al., 2013; Sanz, 2010). Among different dietary components, fiber is associated to positive effects on gut microbiota and related metabolome (De Angelis et al., 2015; Sonnenburg et al., 2016; Vitaglione et al., 2015). Fibers are notably reduced in the Western diet (high in fat and simple carbohydrates, low in fibers), compared to Mediterranean diet. Overall, GFD is characterized by lower content of dietary fibers and resistant starch, compared to standard gluten-containing diet (De Palma et al., 2009; Kinsey et al., 2008; Miranda et al., 2014). Compared to a standard gluten-containing diet, healthy subjects being on GFD showed lower level of LactobacillusBifidobacterium, along with higher number of Enterobacteriaceae (De Palma et al., 2009; Golfetto et al., 2014; Uy et al., 2015). In addition, GFD decreased the production of pro-inflammatory cytokines and chemokines (TNF- $\alpha$, interferon- $\gamma$ and IL-8) and anti-inflammatory cytokines (IL-10) by peripheral blood mononuclear cells (De Palma et al., 2009). The dysbiosis and the modification of the immunological properties suggest that CD children under GFD (T-CD) may lead to increased health risks in T-CD (Sanz, 2010; Galipeau et al., 2015).

Based on the key-role played by gut microbiota in human health, this review aims at describing the most recent advances about oral and fecal microbiota and metabolome of celiac children under GFD.

\section{Salivary microbiota and metabolome in T-CD children}

Human salivary microbiota is composed of ca. 700 bacterial species, reaching cell density of ca. $11 \log$ CFU/g of wet weight dental plaque and 8-9 $\log$ CFU/g of saliva (Maukonen et al., 2008). Several bacterial species are involved in oral diseases, such as dental caries and periodontitis (Bik et al., 2010; Ling et al., 2010). In addition, non-oral diseases such as bacterial endocarditis (Lockhart and Durak, 1999), heart disease (Beck et al., 2005), obesity (Piombino et al., 2014), pneumonia (Paju and Scannapeico, 2007), atherosclerosis (Koren et al., 2011) and preterm low birth weight (Boggess et al., 2006) are also correlated with several oral bacteria. Saliva has recently been considered as a new tool for diagnosis of some diseases (Zarco et al., 2012).

\subsection{The salivary microbiota associated to $T-C D$ children}

Compared to healthy individuals ( $\mathrm{HC}$ ), the salivary microbiota of TCD children is different (Acar et al., 2012; Ercolini et al., 2015; Francavilla et al., 2014). Based on culture-dependent methods, T-CD children were characterized by the lowest prevalence of salivary mutans streptococci and lactobacilli (Acar et al., 2012) and total anaerobes, and by an increased level of Enterobacteriaceae (Francavilla et al., 2014). Analyzing community-level catabolic profiles, the lowest values of Shannon's diversity and substrate richness were found in T-CD children (Francavilla et al., 2014). Consistently, 16S rRNA gene-based metagenetics data showed the lowest values of richness estimator (Chao1) and diversity index (Shannon) in the saliva of T-CD children. The relative abundance of several operational taxonomic units (OTUs) differed between the salivary samples of T-CD children and those of HC. Within Firmicutes, Lachnospiraceae, Gemellaceae, and Streptococcus sanguinis were mainly associated with T-CD children. Streptococcus thermophilus markedly decreased in T-CD children compared to HC. Other Firmicutes (e.g., Veillonella parvula), associated with oral health (Kumar et al., 2005), were found at the lowest relative amount in the saliva of T-CD (Francavilla et al., 2014). Compared to HC, T-CD children showed a decreased level of Bacteroidetes (such as Porphyromonas sp., Porphyromonas endodontalis, and Prevotella nanceiensis), and a lower amount of Actinobacteria, Actinomyces, Atopobium and Corynebacterium durum. Rothia mucilaginosa, involved in gluten degradation (Fernandez-Feo et al., 2013; Zamakhchari et al., 2011), was the only Actinobacteria species higher in T-CD compared to HC children (Francavilla et al., 2014).

Recently, the salivary microbiota of fourteen T-CD Saharawi children, with biopsy-confirmed $C D$, and being on an African-style diet was studied (Ercolini et al., 2015). Saharawi T-CD children showed alpha-diversity indexes similar to the Italian T-CD children. However, Saharawi T-CD children showed an unusually high level of some Firmicutes (Clostridium, Eubacterium, Mogibacterium, Catonella, Peptococcus, Filifactor, Peptostreptococcus), Actinobacteria (Actinomyces, Rothia) and Tenericutes (Bulleidia) compared to Italian T-CD and HC children (Fig. 1) (Ercolini et al., 2015; Francavilla et al., 2014).

A characterization of the salivary microbiota of $U-C D$ patients at the diagnosis is needed to understand the role of the salivary microbiota in the etiology of $C D$. Studies dealing with different severities of $C D$ may be useful to determine the correlations between the severity of $C D$ and the salivary microbiome.

\subsection{Diet drives the salivary microbiota of $T-C D$ children}

In a recent attempt to understand the effect of westernization, Saharawi T-CD children were moved to Italy and subjected, for two months, to shift from the traditional African-style GFD to the Italianstyle GFD (Ercolini et al., 2015). Compared to Italian-style GFD, African-style diet was richer in gluten-free cereals, legumes and vegetables, with a lower intake of animal proteins, sugars, starch and fat. Compared to African-style GFD, the relative abundance of Granulicatella, Capnocytophaga, Porphyromonas and Neisseria was higher after 30 and, especially, 60 days of Italian-style GFD, altering the 'salivary type' of the individuals (Fig. 1). As shown by partitioning around medoid (PAM) clustering, using the relative abundance of core genera, Italian HC and T-CD and Saharawi T-CD children salivary samples were grouped into three "salivary types" (Ercolini et al., 2015). The salivary 


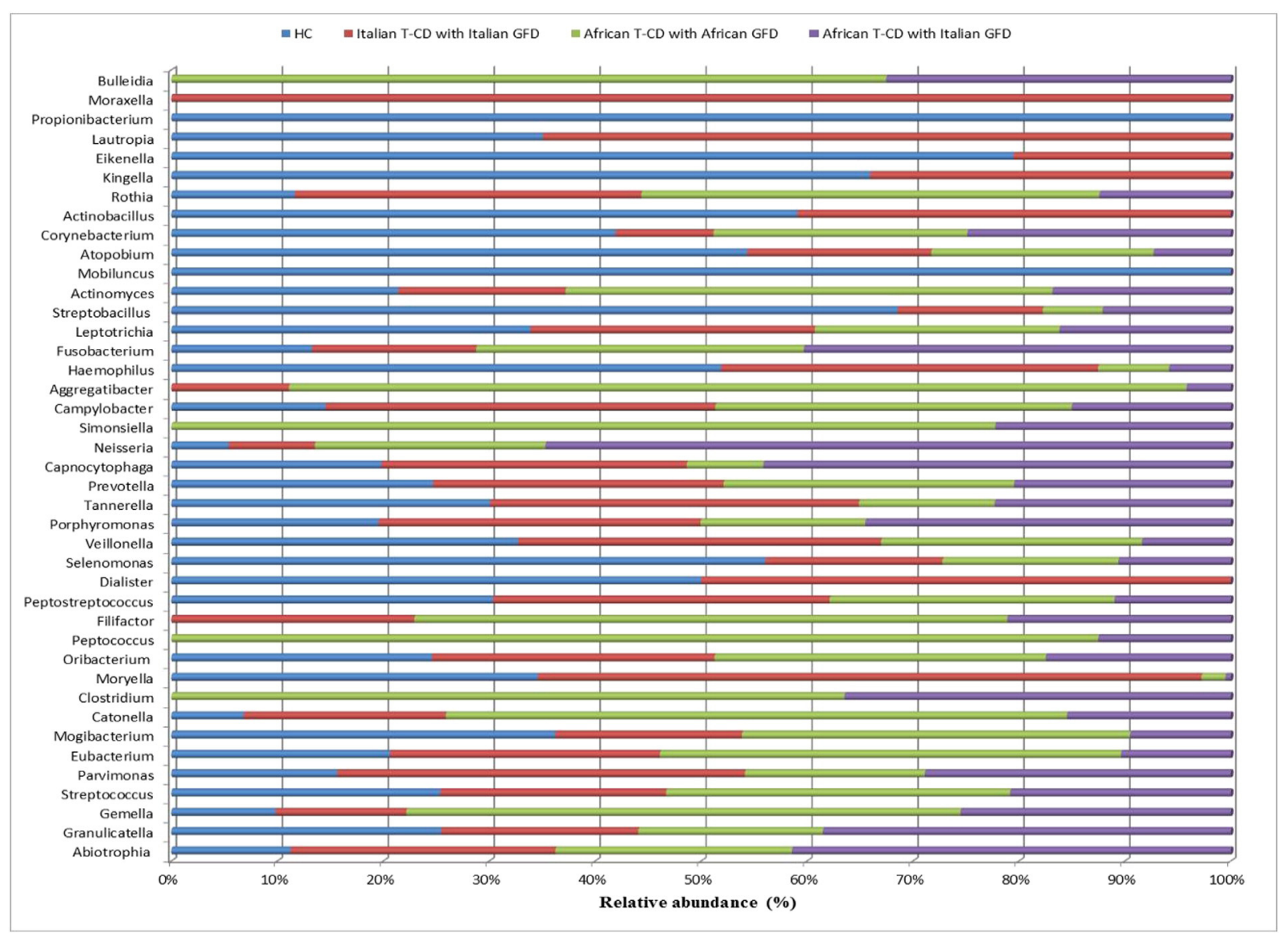

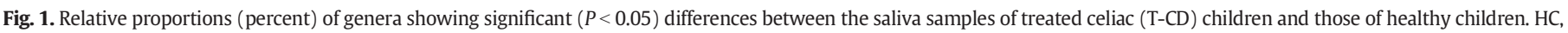

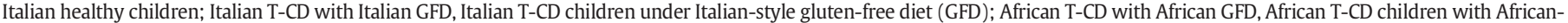

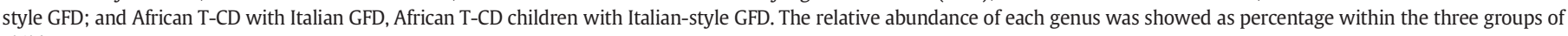
children.

samples of Saharawi T-CD children were grouped in the clusters II (mainly grouping samples collected after 30 and 60 days of Italianstyle GFD) and III (mainly grouping samples collected during Africanstyle GFD), which were associated to the highest amount of Porphyromonas, Neisseria and Granulicatella and Streptococcus, Actinomyces, Peptococcus and Clostridia, respectively. OTU co-occurrence/exclusion patterns showed that the initial equilibrium of co-occurring microbial species was modified by a change in diet, leading to a decrease of the microbial diversity, with a few OTUs out-competing the previously established microbiota and becoming dominant. The abundance of several OTUs (e.g., gluten-degrading Clostridium and Rothia species) can be attributed to environmental and dietary factors, as suggested by the decrease of those OTUs following the period wherein the Saharawi children were on Italian-style GFD. Several correlations were found between OTUs and dietary components. Veionella, Streptococcus, Catonella, Mogibacterium, Clostrium, Peptococcus, Gemella and Actinobacteria showed positive correlations with dietary components (e.g., fiber) mainly abundant in the African-style GDF. Bacteroidetes, Porphyromonas, and Capnocytophaga were negatively correlated with carbohydrates and fibre intake and positively correlated with proteins, iron, calcium, phosphorus, caloric intake and/or lipids. A similar trend was also found for Neisseria and Granulicatella (Ercolini et al., 2015).

Compared to HC, saliva samples of U-CD and T-CD patients showed a significant difference in buffer capacity, IgA levels, minute volume, calcium and $\mathrm{Ca} / \mathrm{P}$ ratio and protein composition (Mina et al., 2008, 2012). This different composition of the saliva in T-CD patients could modify the oral environment and, consequently, the composition and activity of the microbiota.

\subsection{Salivary metabolome associated to $T$-CD children}

Salivary metabolome was analyzed for different human healthy aspects: physiology, diagnostics, functional genomics, pharmacology, toxicology, and nutrition (Winder et al., 2011; Zhang et al., 2012). The salivary metabolome is originated by compounds from different origins, such as environmental exposure through inhalation, food intake, transdermal absorption and metabolic activities of oral microbiota. Regarding the latter, the metabolic products by microbiota interact with human cells (Want et al., 2007) and may also affect the total metabolomic profile (AL-Kateb et al., 2013). Habitual diet was suggested to have an influence on the composition of organic metabolites found in the saliva of healthy adults (De Filippis et al., 2014), again highlighting that diet has a pivotal role in the oral physiology even in healthy individuals. Recently, saliva metabolome was studied in T-CD children (Francavilla et al., 2014; Ercolini et al., 2015). According to the composition of the microbiota, the level of salivary volatile organic compounds (VOC) differed between T-CD and HC children (Fig. 2) (Francavilla et al., 2014). The level of several alcohols and phenols (e.g., 2-ethyl-1-hexanol, 4-1,1,3,3-tetramethylbutyl-phenol, and ethyl alcohol), hydrocarbons (e.g., 1-octadecene), ketones, terpenes, butanoic acid, acetic acid ethyl ester and octanal were the highest in salivary samples of HC. On the contrary, nonanal, 2-methyloctyl ester, carbone disulfide, halogenated and aromatic hydrocarbons (e.g., 1-chlorodecane and trichloromethane) were associated to T-CD children. Previously, increased levels of 2-propyl-1-pentanol, nonanal, dihydro-4-methyl$2(3 \mathrm{H})$-furanone and nonanoic acid were found in breath of HC subjects under GFD, compared to gluten-containing diet (Baranska et al., 2013). 


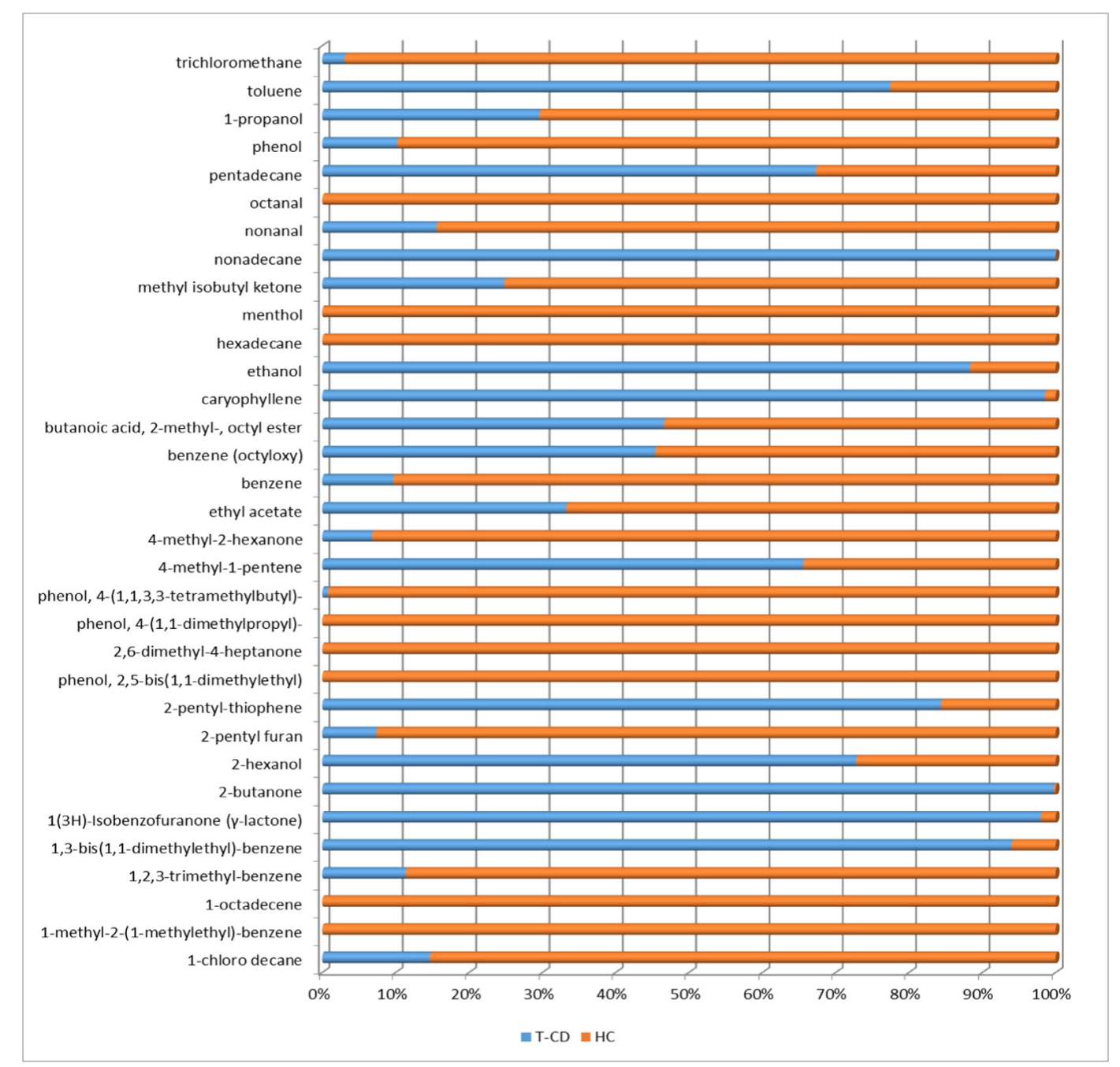

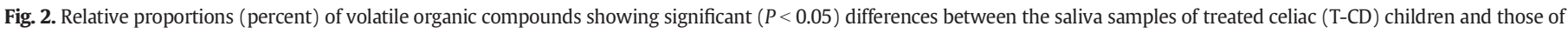
healthy children $(\mathrm{HC})$. The relative abundance of each compound was showed as percentage within the three groups of children.

Salivary microbiota and VOC were variously correlated (Francavilla et al., 2014). In detail, Prevotella sp. was positively correlated with the levels of nonanal and 1-chlorodecane. C. durum was positively associated to the level of 1,2,3-trimethylbenzene, 2,6-dimethyl-4-heptanone, 4methyl-2- hexanone, and 4-methyl-3-penten-2-one. G. adiacens, Atopobium sp., and Bacilli and V. parvula, S. thermophilus, and the division SR1 showed correlations with the amounts of $1(3 \mathrm{H})$ isobenzofuranone (-lactone) and 1-octadecene, and $\gamma$-lactone and 1chlorodecane, respectively. The above data support the hypothesis that the composition of the oral metabolome can be affected by the GFD and composition of the microbiome. The salivary metabolome of Saharawi T-CD children strongly differed from Italian HC and T-CD children (Fig. 3). Several VOC, such as 1-propanol, 4-(1,1,3,3tetramethylbutyl)-phenol and phenol, heptanal, octanal and nonanal, 2-pentyl furan 1,3-bis 1,1-dimethylethyl-benzene; 1-chlorodecane; 1octadecene, benzene and trichloromethane, strongly decreased in Saharawi celiac children during Italian-style GFD. At the same time, the amount of other VOC (e.g., acetone, 2-butanone and 3-methylbutanone) increased under Italian-style GFD. Analysis (by PICRUSt) of predicted metagenomes of the salivary microbiome of the Saharawi T-CD children showed a remarkable modification of the metabolic potential of the microbiome following the diet change, with increased amino acid, vitamin and co-factor metabolisms during Italian-style GFD (Ercolini et al., 2015). In addition, several positive and negative correlations were detected between the relative abundances of several OTUs and VOCs (Fig. S1). For instance, Actinomyces, showing the highest level in saliva samples of Saharawi T-CD on an African-style GFD, showed positive correlations with VOC detected at 0 day of Italianstyle GFD and negative correlations with other VOC detected at 60 days of Italian-style GFD (Fig. S1 panel T0 vs T60). Other OTUs that were dominant during African-style GFD showed negative correlation with VOC associated to Italian-style GFD (e.g., Clostridium versus furan, and Eubacterium versus ethyl acetate) (Fig. S1 panel T30). Salivary metabolome of African T-CD children was also affected by the dietary components of GFD (Fig. S2). For instance, furans, acetone, ethyl acetate, 2butanone and 3-methyl-2-butanone were positively correlated with the intake of proteins. As expected, acetone was also strongly associated to the intake of lipids. Based on this data, dietary components and the initial salivary microbiota drive the salivary metabolome, which, in turn, modifies the colonization and balance of other bacteria (Ercolini et al., 2015).

All together the above data showed that $\mathrm{CD}$ patients have a different salivary microbiota and, especially, metabolome compared to those of healthy control. The limitation of the above studies was related to the small number of $C D$ patients analyzed. However, the findings of these studies support the need of more extensive analyses to define how salivary microbiota/metabolome could impact CD or whether it is just a marker of health status in these individuals. 


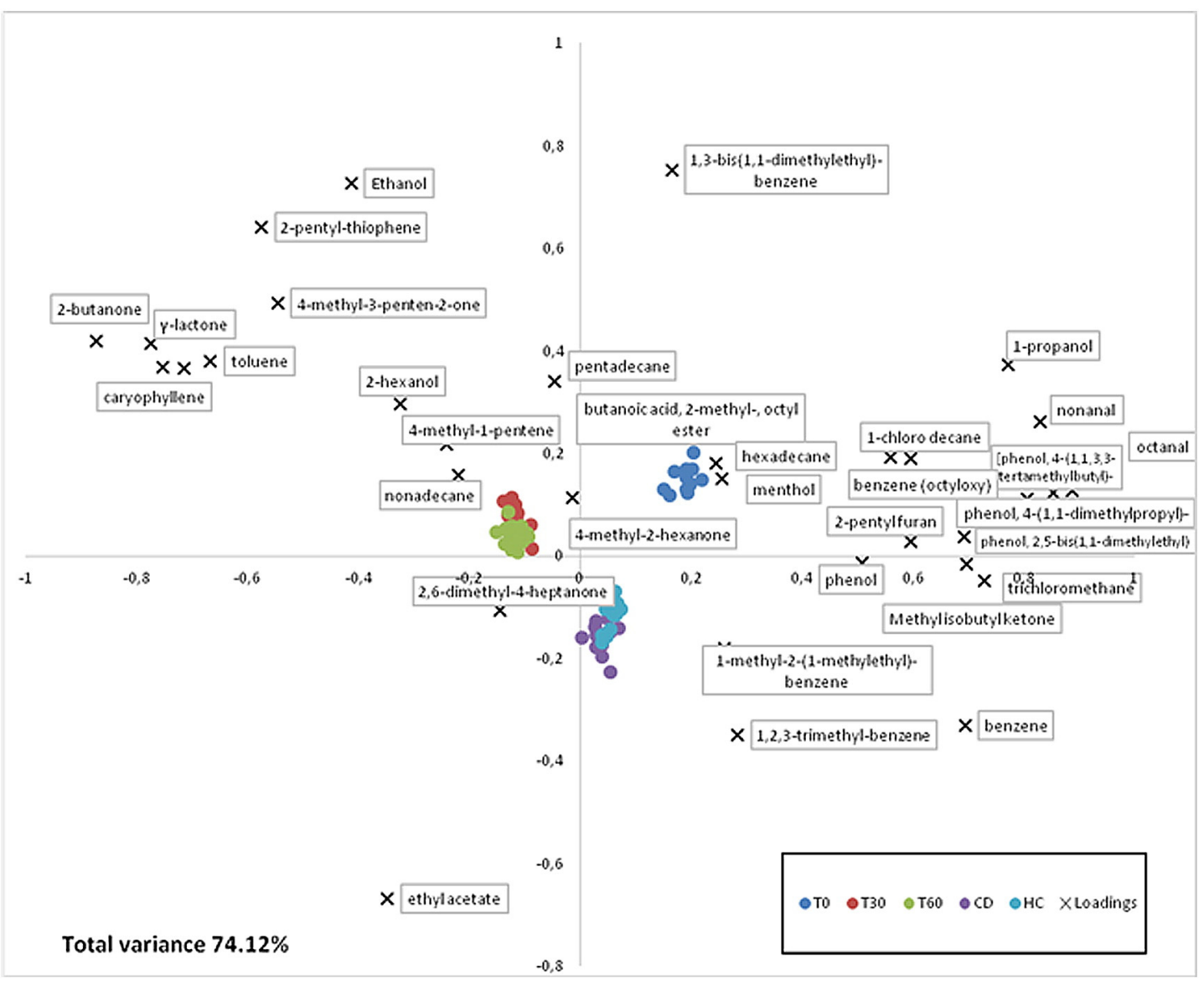

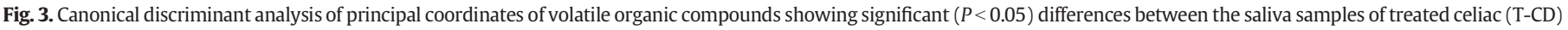

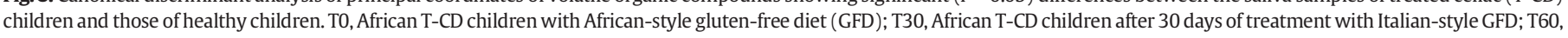
African T-CD children after 60 days of treatment with Italian-style GFD; CD, Italian T-CD children under Italian-style GFD; HC, Italian healthy children under gluten-content diet.

\section{Intestinal microbiota and metabolome of T-CD children}

The intestinal tract is the broadest body-environment interface, where various external materials, such as food components (including allergens) and bacteria (including pathogens), interact with the human body (Verdu et al., 2015). The human intestinal microbiota start gut colonization from the birth and reach levels up to 12 log of bacteria/g of colon (Zoetendal et al., 2012). Bacteria have intimate relationship with the human gut, conditioning the healthy or diseased state by metabolic activities and affecting nutrient absorption, barrier functions and immune development. The integrity of the intestinal microbiota is essential for maintaining a healthy state (Schippa and Conte, 2014). Apart from gluten, additional environmental factors that affect the CD pathogenesis could involve specific bacterial components (Verdu et al., 2015).

\subsection{Intestinal microbiota associated to $T$-CD children}

Different studies showed bacterial dysbiosis at duodenal and/or fecal level of CD patients with active untreated (U-CD; celiac subjects under gluten-containing diet) or treated (T-CD; celiac subjects under GFD for at least two years) disease, compared to healthy subjects (Fig. 4). Duodenal biopsy specimens of U-CD or T-CD children showed the highest abundance of Gram-negative bacteria (Collado et al., 2009; Di Cagno et al., 2011; Nadal et al., 2007; Nistal et al., 2012a; Sánchez et al., 2010, 2013; Schippa et al., 2010). Compared to HC, duodenal biopsies of U-CD showed higher levels of Neisseria, Streptococcus, Serratia and Haemophilus (Cheng et al., 2013; Ou et al., 2009). T-CD patients with persistent CD symptoms showed higher levels of Proteobacteria (e.g., Actinobacter and Neisseria) compared to HC (Wacklin et al., 2014). Overall, the GFD treatment did not completely restore the normal intestinal microbiota (for reviews see de Sousa Moraes et al., 2014; Marasco et al., 2016; Verdu et al., 2015). The relative abundance of Bacteroides, Escherichia coli, Enterobacteriaceae, and Staphylococcus groups was higher in the U-CD than in the healthy subjects (Collado et al., 2009; Nadal et al., 2007; Sánchez et al., 2010, 2013). The ratio of Lactobacillus-Bifidobacterium, and Bacteroides-Enterobacteriaceae was lower in U-CD and T-CD compared to healthy subjects (Di Cagno et al., 2011; Nadal et al., 2007; Schippa et al., 2010). In addition, different balance of Bifidobacterium species was found in the duodenal biopsies of U-CD and T-CD compared to HC (Collado et al., 2008). The amount of some Staphylococcus and Enterococcus species also differed in fecal samples of U-CD and T-CD compared to HC (Sánchez et al., 2012a). The relative amount of Bacteroides was higher in U-CD and/or T-CD than in healthy children (Collado et al., 2007, 2009; Di Cagno et al., 2011; Nadal et al., 2007; Sánchez et al., 2010; Schippa et al., 2010). However, Bacteroides is a dominant genus of human gut microbiota and only some species (e.g., B. vulgatus, B. fragilis) show pro-inflammatory effect (Setoyama et al., 2003), suggesting the importance of identification at the species level (de Sousa Moraes et al., 2014; Sánchez et al., 2012b). Compared to healthy subjects, the relative abundance of Bacteroides-Prevotella increased in U-CD and T-CD children, along with a reduction of IgA-coated bacteria, suggesting the existence of mucosal barrier defects in CD patients (De Palma et al., 2010a). The gut microbiota could affect the pathogenesis and progression of $C D$ by activation of innate immune system, modulation of the epithelial barrier and/or exacerbation of the immune response to gliadin peptides (Verdu et al., 2015; Galipeau et al., 2015). 


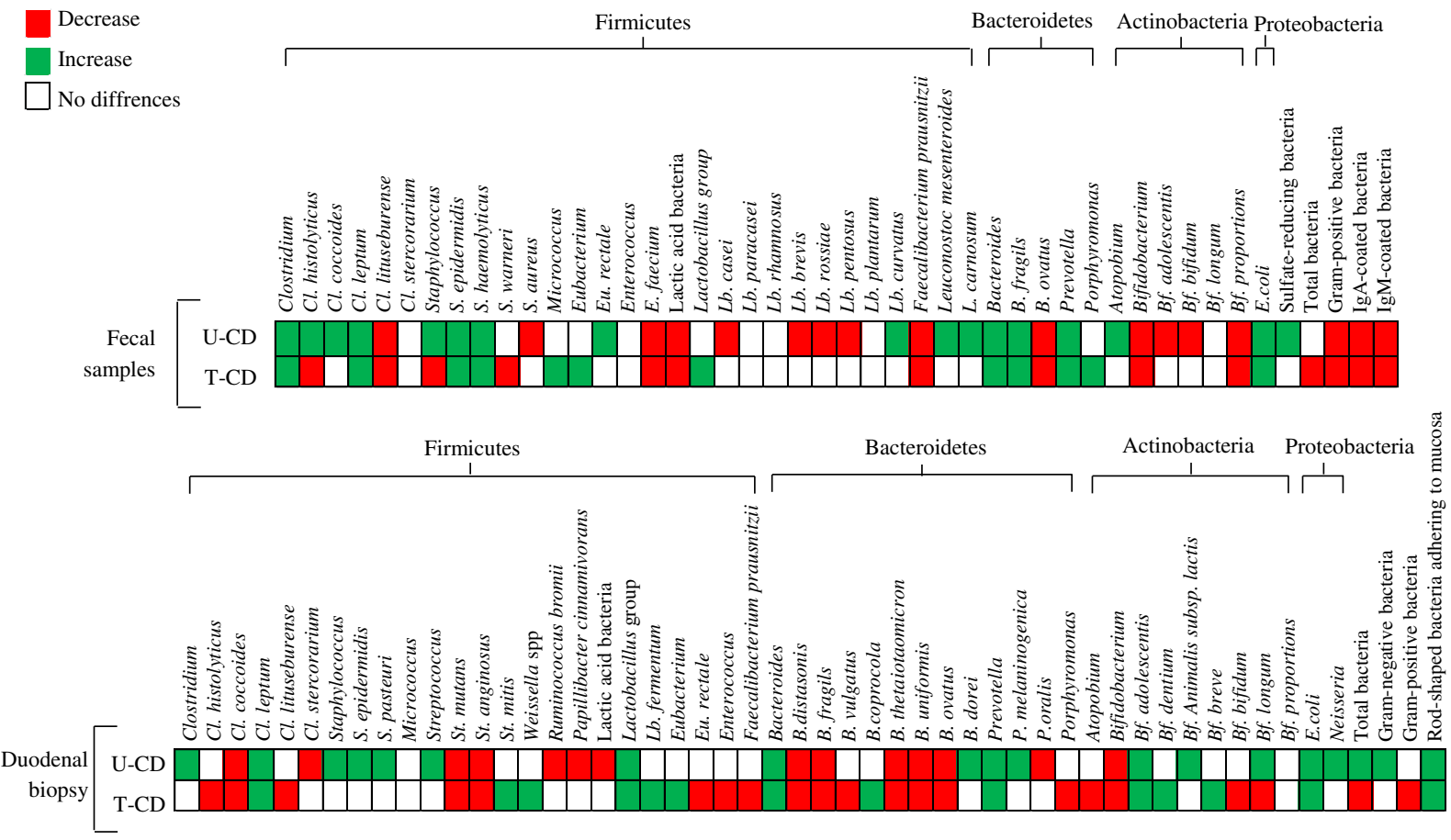

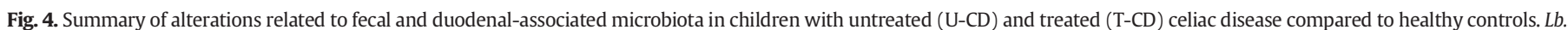

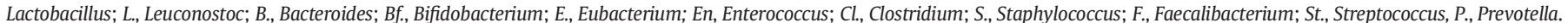

Various studies suggested that genetics and gut microbiota play a role in the susceptibility to $\mathrm{CD}$. Compared to infants with low genetic risk of CD, Bacteroides-Prevotella group, Gram-negative bacteria, Streptococcus-Lactococcus, Eubacterium rectale, Clostridium coccoides, Clostridium lituseburense, Clostridium histolyticum and sulfite-reducing bacteria were higher in infants with high genetic risk of CD (De Palma et al., 2010a, 2010b; Olivares et al., 2014). High level of Bacteroides was found also in infants with high genetic risk of CD (Sánchez et al., 2011). In addition, a study with large cohort of infants showed low level of Bifidobacterium and increase of Bacteroides fragilis and Staphylococcus associated with infants with high genetic risk of CD (de Palma et al., 2012).

However, consensus across studies to define specific bacterial genera and/or species in active CD or T-CD patients is still lacking (Verdu et al., 2015). Several variables and confounding factors (e.g., age, methodology, sampling technique, length of GFD and clinical conditions) make the comparison of different studies hard. Although CD is common to children and adults (Vivas et al., 2008), only few studies are available for the intestinal composition of U-CD and T-CD adults (Nistal et al., 2012a, 2012b). To determine the exact relationship between gut microbiota and CD, further studies including CD adults are necessary (de Sousa Moraes et al., 2014; Verdu et al., 2015).

\subsection{Serum, fecal and urinary metabolome associated to T-CD children}

Compared to healthy controls, U-CD and T-CD patients showed unbalanced serum, fecal and urine metabolome (Di Cagno et al., 2009, 2011; Bertini et al., 2009). U-CD patients showed the lowest levels of serum amino acids, lipids, pyruvate and choline, and the highest levels of glucose and 3-hydroxybutyric acid (Bertini et al., 2009). The modified levels of glucose and ketones suggest alterations of energy metabolism in U-CD patients. After 12 months of GFD, an improved glucose metabolism, a decrease of 3-hydroxybutyric acid and an increase of methionine, valine, lactate and creatinine were found in the urine (Bertini et al., 2009). In agreement with gut dysbiosis, urines from U-CD and T-CD patients showed altered levels of some microbial metabolites, such as indoxyl sulfate, meta-[hydroxyphenyl]propionic acid and phenylacetylglycine (Bertini et al., 2009). Median values of Lys, Arg, creatine and methylamine were higher in T-CD than in HC children (Di Cagno et al., 2011). On the contrary, carnosine, glucose, glutamine and 3-methyl-2-oxobutanoic acid were the highest in HC children. In addition, fecal levels of Trp, Pro, Asn, His, Met, trimethylamine-N-ox and tyramine were higher in T-CD than HC children. The level of total esters in fecal and urine samples was higher in HC compared to T-CD children, but several of them (ethyl-acetate, octyl-acetate, propylbutyrate, propyl-propanoate and butyl 2-methylbitanoate) were the highest in fecal samples of T-CD (Di Cagno et al., 2011). Esterification reactions in colon could be the microbial strategy to detoxify acids or alcohols (Vitali et al., 2010). Aldehydes were higher in HC children than in T-CD (Di Cagno et al., 2011). According to salivary metabolome, some alcohols (e.g., 1-octen-3-ol, ethanol and 1-propanol) were found at the highest level in T-CD. Ethanol synthesized by intestinal bacteria was correlated with non-alcoholic steatohepatitis (NASH) (Cani et al., 2008), which is associated to occult CD (Grieco et al., 2001). Short chain fatty acids (SCFA) (e.g., isocaproic, acetic, butyric and propionic acids) differed in fecal samples of U-CD and T-CD children compared to healthy children (Di Cagno et al., 2009, 2011; Tjellström et al. 2005). The altered composition of the metabolome of U-CD and T-CD subjects could be related to $\mathrm{CD}$, as well as to bacterial dysbiosis and composition of GFD. Several bacterial metabolites (e.g., acetic acid, propionic acid and butyric acid) and structures (e.g., polysaccharide $A$ of $B$. fragilis) could modify the immune responses of CD patients (Verdu et al., 2015).

It is widely accepted that microbial metabolites probably act as mediators for the host metabolism with positive (e.g., butyrate) or detrimental (e.g., trimethylamine-N-ox) effects (Sonnenburg and Bäckhed, 2016). In the same time, the production of VOCs and other metabolites is affected by complex interactions between host, composition of microbiota and diet. Consequently, meta-genomics, meta-trascriptomic and/ or meta-proteomics studies with the reconstruction of metabolic pathways could be the best tool to elucidate the relationship between microbiota and metaboloma, microbiota and immune function, and metaboloma and immune function in $\mathrm{CD}$ patients. 


\section{Concluding remarks and future perspectives}

Although not yet exhaustive, the current literature clearly shows the association between $\mathrm{CD}$, gut microbiota and metabolome. Altered gut microbiota and metabolome could play a secondary role in aggravating $\mathrm{CD}$ pathogenesis or other diseases in celiac patients by modifying the host immunity and physiology. CD patients at the diagnosis, when still consuming a gluten-containing diet, showed different microbiota compared to healthy controls, suggesting that the microbiota is involved the CD pathogenesis (Cenit et al., 2015). Another hypothesis is that CD can promote dysbiosis, which, in turn, can increase the inflammation by pro-inflammatory pathobionts (e.g. strains belonging to Enterobacteriaceae, $B$. fragilis) and decline of anti-inflammatory mutualistic bacteria (e.g. strains belonging to Lactobacillus and Bifidobacterium). Further studies linking bacterial dysbiosis and development of CD in high-risk infants will help to define the gene-microbe interactions and the role of the microbiota on the $\mathrm{CD}$ pathogenesis and progression (Verdu et al., 2015; Galipeau et al., 2015).

However, consensus across studies defining specific bacteria and metabolites in children and adult celiac patients is still lacking. In addition, GFD could also affect the gut microbiota and, consequently, the metabolome of celiac patients (De Palma et al., 2009; Bonder et al., 2016). To date, studies were performed only comparing the microbiota and metabolome of T-CD patients under GFD with those of healthy volunteers under gluten-containing diet. Wheat is the major source of fructans in the diet, and contains $1-4 \%$ fructans on solid matter (Gibson and Shepherd, 2010). Intake of prebiotic fructans (oligofructose and inulin) resistant starch and dextrin from cereals during glutencontaining diet promotes growth of anti-inflammatory mutualistic bacteria (Slavin, 2013). Further studies are strongly recommended to elucidate the effect exerted by certain compounds contained in cereals on the gut microbiota. This could be done by comparing T-CD patients to healthy subjects under GFD.

Data on the use of probiotics (e.g., bifidobacteria) for CD are encouraging, but to date only a few studies (de Sousa Moraes et al., 2014) have been conducted on humans, also with contradictory results. For probiotics, the complexity of the microbiome and the intra and intersubject variability further complicate the definition of what a desired state may look like for a population or for a given individual. By contrast, a growing interest is assuming the quality and preparation of glutenfree foods. Emerging dietary treatments are not only economical, but also offer a targetable and non-invasive approach for chronic states of inflammation. After long-time work, our group developed a biotechnology strategy aimed at hydrolyzing gluten $(<8 \mathrm{ppm}$ of residual concentration) during food processing with a combination of selected sourdough lactobacilli and food-grade fungal proteases (Greco et al., 2011). Conceptually, the only dietary difference between CD and healthy individuals (gluten or similar protein-containing flours) was eliminated. In conclusion, dietary strategies could improve the intestinal microbiota and host immunity increasing the resilience to $C D$.

\section{Appendix A. Supplementary data}

Supplementary data to this article can be found online at http://dx. doi.org/10.1016/j.ijfoodmicro.2016.07.025.

\section{References}

Acar, S., Yetkiner, A.A., Ersin, N., Oncag, O., Aydogdu, S., Arikan, C., 2012. Oral findings and salivary parameters in children with celiac disease: a preliminary study. Med. Princ Pract. 21, 129-133.

Albenberg, L.G., Wu, G.D., 2014. Diet and the intestinal microbiome: associations, functions, and implications for health and disease. Gastroenterology 146, 1564-1572.

AL-Kateb, H., de Lacy Costello, B., Ratcliffe, N., 2013. An investigation of volatile organic compounds from the saliva of healthy individuals using headspace-trap/GC-MS. J. Breath Res. 7, 036004

Baranska, A., Tigchelaar, E., Smolinska, A., Dallinga, J.W., Moonen, E.J., Dekens, J.A., Wijmenga, C., Zhernakova, A., van Schooten, F.J., 2013. Profile of volatile organic compounds in exhaled breath changes as a result of gluten-free diet. J. Breath Res. 7, 037104

Beck, J.D., Eke, P., Heiss, G., Manadios, P., Couper, D., Lin, D., Moss, K., Elter, J., Offenbacher, S., 2005. Periodontal disease and coronary heart disease: a reappraisal of the exposure. Circulation 112, 19-24.

Bertini, I., Calabro, A., De Carli, V., Luchinat, C., Nepi, S., Porfirio, B., Renzi, D., Saccenti, E., Tenori, L., 2009. The metabonomic signature of celiac disease. J. Proteome Res. 8, 170-177.

Bik, M.E., Long, C.D., Armitage, G.C., Loomer, P., Emerson, J., Mongodin, E.F., Nelson, K.E., Gill, S.R., Fraser-Liggett, C.M., Relman, D.A., 2010. Bacterial diversity in the oral cavity of ten healthy individuals. ISME J. 4, 962-974.

Boggess, K.A., Beck, J.D., Murtha, A.P., Moss, K., Offenbacher, S., 2006. Maternal periodontal disease in early pregnancy and risk for a small-forgestational-age infant. Am. J. Obstet. Gynecol. 194, 1316-1322.

Bonder, M.J., Tigchelaar, E.F., Cai, X., Trynka, G., Cenit, M.C., Hrdlickova, B., Zhong, H. Vatanen, T., Gevers, D., Wijmenga, C., Wang, Y., Zhernakova, A., 2016. The influence of a short-term gluten-free diet on the human gut microbiome. Genome Med. 8, 45.

Cani, P.D., Bibiloni, R., Knauf, C., Waget, A., Neyrinck, A.M., Delzenne, N.M., Burcelin, R., 2008. Changes in gut microbiota control metabolic endotoxemia-induced inflammation in high-fat diet-induced obesity and diabetes in mice. Diabetes 57, 1470-1481.

Caputo, I., Lepretti, M., Martucciello, S., Esposito, C., 2010. Enzymatic strategies to detoxify gluten: implications for celiac disease. Enzym. Res. 2010, 174354.

Cenit, M.C., Olivares, M., Codoñer-Franch, P., Sanz, Y., 2015. Intestinal microbiota and celiac disease: cause, consequence or co-evolution? Nutrients 7, 6900-6923.

Cheng, J., Kalliomäki, M., Heilig, H.G., Palva, A., Lähteenoja, H., de Vos, W.M., Salojärvi, J., Satokari, R., 2013. Duodenal microbiota composition and mucosal homeostasis in pediatric celiac disease. BMC Gastroenterol. 13, 113.

Claesson, M.J., Jeffery, A.B., Conde, S., Power, S.E., O'Connor, E.M., Cusack, S., Harris, H.M.B., Coakley, M., Lakshminarayanan, B., O'Sullivan, O., Fitzgerald, G.F., Deane, J., O'Connor, M., Harnedy, N., O'Connor, K., O'Mahony, D., van Sinderen, D., Wallace, M., Brennan, L., Stanton, C., Marchesi, J.R., Fitzgerald, A.P., Shanahan, F., Hill, C., Ross, R.P., O'Toole, P.W., 2012. Gut microbiota composition correlates with diet and health in the elderly. Nature 488, 178.

Collado, M.C., Calabuig, M., Sanz, Y., 2007. Differences between the fecal microbiota of coeliac infants and healthy controls. Curr. Issues Intest. Microbiol. 8, 9-14.

Collado, M., Donat, E., Ribes-Koninckx, C., Calabuig, M., Sanz, Y., 2008. Imbalances in faecal and duodenal Bifidobacterium species composition in active and non-active coeliac disease. BMC Microbiol. 8, 232

Collado, M.C., Donat, E., Ribes-Koninckx, C., Calabuig, M., Sanz, Y., 2009. Specific duodenal and faecal bacterial groups associated with paediatric coeliac disease. J. Clin. Pathol. 62, 264-269.

De Angelis, M., Cassone, A., Rizzello, C.G., Gagliardi, F., Minervini, F., Calasso, M., Di Cagno, R., Francavilla, Gobbetti, M., 2010. Mechanism of degradation of immunogenic gluten epitopes from Triticum turgidum L. var. durum by sourdough lactobacilli and fungal proteases. Appl. Environ. Microbiol. 76, 508-518.

De Angelis, M., Montemurno, E., Vannini, L., Cosola, C., Cavallo, N., Gozzi, G., Maranzano, V., Di Cagno, R., Gobbetti, M., Gesualdo, L., 2015. The role of whole-grain barley on human fecal microbiota and metabolome. Appl. Environ. Microbiol. 81, 7945-7956.

De Filippis, F., Vannini, L., La Storia, A., Laghi, L., Piombino, P., Stellato, G., Serrazanetti, D.I., Gozzi, G., Turroni, S., Ferrocino, I., Lazzi, C., Di Cagno, R., Gobbetti, M., Ercolini, D., 2014. The same microbiota and a potentially discriminant metabolome in the saliva of omnivore, ovo-lacto-vegetarian and vegan individuals. PLoS One 9, e112373.

De Palma, G., Cinova, J., Stepankova, R., Tuckova, L., Sanz, Y., 2010a. Pivotal advance: bifidobacteria and Gram-negative bacteria differentially influence immune responses in the proinflammatory milieu of celiac disease. J. Leukoc. Biol. 87, 765-778.

De Palma, G., Kamanova, J., Cinova, J., Olivares, M., Drasarova, H., Tuckova, L., Sanz, Y., 2012. Modulation of phenotypic and functional maturation of dendritic cells by intestinal bacteria and gliadin: relevance for celiac disease. J. Leukoc. Biol. 92, 1043-1054.

De Palma, G., Nadal, I., Collado, M.C., Sanz, Y., 2009. Effects of a gluten-free diet on gut microbiota and immune function in healthy adult human subjects. Br. J. Nutr. 102, 1159.

De Palma, G., Nadal, I., Medina, M., Donat, E., Ribes-Koninckx, C., Calabuig, M., Sanz, Y., 2010b. Intestinal dysbiosis and reduced immunoglobulin-coated bacteria associated with coeliac disease in children. BMC Microbiol. 10, 63.

Di Cagno, R., De Angelis, M., De Pasquale, I., Ndagijimana, M., Vernocchi, P., Ricciuti, P., Gagliardi, F., Laghi, L., Crecchio, C., Guerzoni, M.E., Gobbetti, M., Francavilla, R., 2011. Duodenal and faecal microbiota of celiac children: molecular, phenotype and metabolome characterization. BMC Microbiol. 11, 219

Di Cagno, R., Rizzello, C.G., De Angelis, M., Cassone, A., Giuliani, G., Banedusi, A., Limitone, A., Surico, R.F., Gobbetti, M., 2008. Use of selected sourdough strains of Lactobacillus for removing gluten and enhancing the nutritional properties of gluten-free bread. J. Food Prot. 71, 1491-1495.

Di Cagno, R., Rizziello, C.G., Gagliardi, F., Ricciuti, P., Ndagijimana, M., Francavilla, R., Guerzoni, M.E., Crecchio, C., Gobbetti, M., De Angelis, M., 2009. Different fecal microbiotas and volatile organic compounds in treated and untreated children with celiac disease. Appl. Environ. Microbiol. 75, 3963-3971.

Di Sabatino, A., Corazza, G.R., 2009. Coeliac disease. Lancet 373, 1480-1493.

Ercolini, D., Francavilla, R., Vannini, L., De Filippis, F., Capriati, T., Di Cagno, R., Iacono, G., De Angelis, M., Gobbetti, M., 2015. From an imbalance to a new imbalance: Italian-style gluten-free diet alters the salivary microbiota and metabolome of African celiac children. Sci. Rep. 18, 18571.

Fasano, A., Catassi, C., 2012. Clinical practice. Celiac disease. N. Engl. J. Med. 367, 2419-2426.

Fernandez-Feo, M., Ewi, G., Blumenjranz, G., Dewhirst, F.E., Schuppan, D., Oppenheim, F.G., Helmerhorst, E.F., 2013. The cultivable human oral gluten-degrading microbiome and its potential implications in celiac disease and gluten sensitivity. Clin. Microbiol. Infect. 19, E386-E394. 
Flint, H.J., Duncan, S.H., Scott, K.P., Louisa, P., 2015. Links between diet, gut microbiota composition and gut metabolism. Proc. Nutr. Soc. 74, 13-22.

Francavilla, R., Ercolini, D., Piccolo, M., Vannini, L., Siragusa, S., De Filippis, F., De Pasquale, I., Di Cagno, R., Di Toma, M., Gozzi, G., Serrazanetti, D.I., De Angelis, M., Gobbetti, M., 2014. Salivary microbiota and metabolome associated with celiac disease. Appl. Environ. Microbiol. 80, 3416-3425.

Galipeau, H.J., McCarville, J.L., Huebener, S., Litwin, O., Meisel, M., Jabri, B., Sanz, Y., Murray, J.A., Jordana, M., Alaedini, A., Chirdo, F.G., Verdu, E.F., 2015. Intestinal microbiota modulates gluten-induced immunopathology in humanized mice. Am. J. Pathol. 185, 2969-2982.

Gibson, P.R., Shepherd, S.J., 2010. Evidence-based dietary management of functional gastrointestinal symptoms: the FODMAP approach. J. Gastroenterol. Hepatol. 25 (2), 252-258.

Gobbetti, M., Rizzello, C.G., Di Cagno, R., De Angelis, M., 2014. How the sourdough may affect the functional features of leavened baked goods. Food Microbiol. 37, 30-40.

Golfetto, L., de Senna, F.D., Hermes, J., Beserra, B.T.S., França, F., Martinello, F., 2014. Lower bifidobacteria counts in adult patients with celiac disease on a gluten-free diet. Arq. Gastroenterol. 51, 139-143.

Greco, L., Gobbetti, M., Auricchio, R., Di Mase, R., Landolfo, F., Paparo, F., Di Cagno, R., De Angelis, M., Rizzello, C.G., Cassone, A., Terrone, G., Timpone, L., D'Aniello, M., Maglio, M., Troncone, R., Auricchio, S., 2011. Safety for patients with celiac disease of baked goods made of wheat flour hydrolyzed during food processing. Clin. Gastroenterol. Hepatol. 9, 24-29.

Grieco, A., Miele, L., Pignataro, G., Pompili, M., Rapaccini, G.L., Gasbarrini, G., 2001. Is coeliac disease a confounding factor in the diagnosis of NASH? Gut 49, 596.

Jeffery, I.B., O'Toole, P.W., 2013. Diet-microbiota interactions and their implications for healthy living. Nutrients 5, 234-252.

Kinsey, L., Burden, S.T., Bannerman, E., 2008. A dietary survey to determine if patients with coeliac disease are meeting current healthy eating guidelines and how their diet compares to that of the British general population. Eur. J. Clin. Nutr. 62, 1333-1342.

Koren, O., Spor, A., Felin, J., Fak, F., Stombaugh, J., Tremaroli, V., Behre, C.J., Knight, R., Fagerberg, B., Ley, R.E., Backhed, F., 2011. Human oral, gut, and plaque microbiota in patients with atherosclerosis. Proc. Natl. Acad. Sci. U. S. A. 108, 4592-4598.

Kumar, P.S., Griffen, A.L., Moeschberger, M.L., Leys, E.J., 2005. Identification of candidate periodontal pathogens and beneficial species by quantitative $16 \mathrm{~S}$ clonal analysis. J. Clin. Microbiol. 43, 3944-3955.

Ling, Z., Kong, J., Jia, P., Wei, C., Wang, Y., Pan, Z., Huang, W., Li, L., Chen, H., Xiang, C., 2010. Analysis of oral microbiota in children with dental caries by PCR-DGGE and barcoded pyrosequencing. Microb. Ecol. 60, 677-690.

Ling, Z., Liu, X., Wang, Y., Li, L., Xiang, C., 2013. Pyrosequencing analysis of the salivary microbiota of healthy Chinese children and adults. Microb. Ecol. 65, 487-495.

Lionetti, P., Favilli, T., Chiaravalloti, G., Ughi, C., Maggiore, G., 1999. Coeliac disease in Saharawi children in Algerian refugee camps. Lancet 353, 1189-1190.

Lockhart, P.B., Durak, D.T., 1999. Oral microflora as a cause of endocarditis and other distant site infections. Infect. Dis. Clin. N. Am. 13, 833-850.

Marasco, G., Di Biase, A.R., Schiumerini, R., Eusebi, L.H., Iughetti, L., Ravaioli, F., Scaioli, E. Colecchia, A., Festi, D., 2016. Gut microbiota and celiac disease. Dig. Dis. Sci. http:// dx.doi.org/10.1007/s10620-015-4020-2.

Maukonen, J., Matto, J., Suihko, M.L., Saarela, M., 2008. Intra-individual diversity and similarity of salivary and faecal microbiota. J. Med. Microbiol. 57, 1560-1568.

Mina, S.S., Azcurra, A.I., Dorronsoro, S., Brunotto, M.N., 2008. Alterations of the oral ecosystem in children with celiac disease. Acta Odontol. Latinoam. 21, 121-126.

Mina, S., Riga, C., Azcurra, A.I., Brunotto, M., 2012. Oral ecosystem alterations in celiac children: a follow-up study. Arch. Oral Biol. 57, 154-160.

Miranda, J., Lasa, A., Bustamante, M.A., Churraca, L., Simon, E., 2014. Nutritional differences between a gluten free diet and diet containing equivalent products with gluten. Plant Foods Hum. Nutr. 69, 182-187.

Mitea, C., Havenaar, R., Drijfhout, J.W., Edens, L., Dekking, L., Koning, F., 2008. Efficient degradation of gluten by a prolyl endoprotease in a gastrointestinal model: implications for coeliac disease. Gut 57, 25-32.

Nadal, I., Donant, E., Ribes-Koninckx, C., Calabuig, M., Sanz, Y., 2007. Imbalance in the composition of the duodenal microbiota of children with coeliac disease. J. Med. Microbiol. 56, 1669-1674.

Nistal, E., Caminero, A., Herrán, A.R., Arias, L., Vivas, S., de Morales, J.M., Calleja, S., de Miera, L.E., Arroyo, P., Casqueiro, J., 2012a. Differences of small intestinal bacteria populations in adults and children with/without celiac disease: effect of age, gluten diet, and disease. Inflamm. Bowel Dis. 18, 649-656.

Nistal, E., Caminero, A., Vivas, S., Ruiz De Morales, J.M., Sáenz De Miera, L.E., RodríguezAparicio, L.B., Casqueiro, J., 2012b. Differences in faecal bacteria populations and faecal bacteria metabolism in healthy adults and celiac disease patients. Biochimie 94 , 1724-1729.

Olivares, M., Neef, A., Castillejo, G., Palma, G.D., Varea, V., Capilla, A., Palau, F., Nova, E., Marcos, A., Polanco, I., Ribes-Koninckx, C., Ortigosa, L., Izquierdo, L., Sanz, Y., 2014. The HLA-DQ2 genotype selects for early intestinal microbiota composition in infants at high risk of developing coeliac disease. Gut 64, 406-417.

Ou, G., Hedberg, M., Hörstedt, P., Baranov, V., Forsberg, G., Drobni, M., Sandström, O., Wai, S.N., Johansson, I., Hammarström, M.L., Hernell, O., Hammarström, S., 2009. Proximal small intestinal microbiota and identification of rod-shaped bacteria associated with childhood celiac disease. Am. J. Gastroenterol. 104, 3058-3067.

Paju, S., Scannapeico, F.A., 2007. Oral biofilm, periodontitis, and pulmonary infections. Oral Dis. $13,508-512$.

Petersen, P.E., 2003. The world oral health report 2003: continuous improvement of oral health in the 21st century-the approach of the WHO Global Oral Health Programme. Community Dent. Oral Epidemiol. 31, 3-23.

Piombino, P., Genevose, A., Esposito, S., Moio, L., Cutolo, P.P., Chambery, A., Severino, V. Moneta, E., Smith, D.P., Owens, S.M., Gilbert, J.A., Ercolini, D., 2014. Saliva from obese individuals suppresses the release of aroma compounds from wine. PLoS One 9, e85611.

Rautava, S., Luoto, R., Salminen, S., Isolauri, E., 2012. Microbial contact during pregnancy, intestinal colonization and human disease. Nat. Rev. Gastroenterol. Hepatol. 9, 565-576.

Sánchez, E., Laparra, J., Sanz, Y., 2012b. Discerning the role of Bacteroides fragilis in celiac disease pathogenesis. Appl. Environ. Microbiol. 78, 6507-6515.

Sánchez, E., Ribes-Koninckx, C., Calabuig, M., Sanz, Y., 2012a. Intestinal Staphylococcus spp. and virulent features associated with coeliac disease. J. Clin. Pathol. 65, 830-834.

Sánchez, E., De Palma, G., Capilla, A., Nova, E., Pozo, T., Castillejo, G., Varea, V., Marcos, A., Garrote, J.A., Polanco, I., Lopez, A., Ribes-Koninchx, Gacria-novo, M.D., Calvo, C., Ortigosa, L., Palau, F., Sanz, Y., 2011. Influence of environmental and genetic factors linked to celiac disease risk on infant gut colonization by Bacteroides species. Appl. Environ. Microbiol. 77, 5316-5323.

Sánchez, E., Donat, E., Ribes-Koninckx, C., Calabuig, M., Sanz, Y., 2010. Intestinal Bacteroides species associated with coeliac disease. J. Clin. Pathol. 63, 1105-1111.

Sánchez, E., Donat, E., Ribes-Koninckx, C., Fernández-Murga, M.L., Sanz, Y., 2013. Duodenal-mucosal bacteria associated with celiac disease in children. Appl. Environ. Microbiol. 79, 5472-5479.

Sanz, Y., 2010. Effects of a gluten-free diet on gut microbiota and immune function in healthy adult humans. Gut Microbes 3, 135-137.

Schippa, S., Conte, M.P., 2014. Dysbiotic events in gut microbiota: impact on human health. Nutrients 6, 5786-5805.

Schippa, S., Iebba, V., Barbato, M., Di Nardo, G., Totino, V., Checchi, M.P., Longhi, C., Maiella, G., Cucchiara, S., Conte, M.P., 2010. A distinctive microbial signature in celiac pediatric patients. BMC Microbiol. 10, 175

Setoyama, H., Imaoka, A., Ishikawa, H., Umesaki, Y., 2003. Prevention of gut inflammation by Bifidobacterium in dextran sulfate-treated gnotobiotic mice associated with Bacteroides strains isolated from ulcerative colitis patients. Microbes Infect. 5, $115-122$.

Slavin, J., 2013. Fiber and prebiotics: mechanisms and health benefits. Nutrients 5 (4) 1417-1435.

Sonnenburg, J.L., Bäckhed, F., 2016. Diet-microbiota interactions as moderators of human metabolism. Nature 6, 56-64.

Sonnenburg, E.D., Smits, S.A., Tikhonov, M., Higginbottom, S.K., Wingreen, S.N. Sonnenburg, J.L., 2016. Diet-induced extinctions in the gut microbiota compound over generations. Nature 529, 212-215.

de Sousa Moraes, L.F., Grzeskowiak, L.M., de Sales Teixeira, T.F., do Carmo Gouveia Peluzio M., 2014. Intestinal microbiota and probiotics in celiac disease. Clin. Microbiol. Rev. 27, 482-489.

Stepniak, D., Koning, F., 2006. Celiac disease-sandwiched between innate and adaptive immunity. Hum. Immunol. 67, 460-468.

Teresi, S., Crapisi, M., Vallejo, M.D., Castellaneta, S.P., Francavilla, R., Iacono, G., Ravelli, A. Menegazzi, P., Louali, M., Catassi, C., 2010. Celiac disease seropositivity in Saharaw children: a follow-up and family study. J. Pediatr. Gastroenterol. Nutr. 50, 506-509.

Tjellström, B., Stenhammar, L., Högberg, L., Fälth-Magnusson, K., Magnusson, K.E. Midtvedt, T., Sundqvist, T., Norin, E., 2005. Gut microflora associated characteristics in children with celiac disease. Am. J. Gastroenterol. 100, 2784-2788.

Uy, N., Graf, L., Lemley, K.V., Kaskel, F., 2015. Effects of gluten-free, dairy-free diet on childhood nephrotic syndrome and gut microbiota. Pediatr. Res. 77, 252-255.

Verdu, E.F., Galipeau, H.J., Jabri, B., 2015. Novel players in coeliac disease pathogenesis: Role of the gut microbiota. Nat. Rev. Gastroenterol. Hepatol. 12, 497-506.

Vitaglione, P., Mennella, I., Ferracane, R., Rivellese, A.A., Giacco, R., Ercolini, D., Gibbons, S.M., La Storia, A., Gilbert, J.A., Jonnalagadda, S., Thielecke, F., Gallo, M.A., Scalfi, L., Fogliano, V., 2015. Wholegrain wheat consumption reduces inflammation in a randomized controlled trial on overweight and obese subjects with unhealthy dietary and lifestyle behaviors: role of polyphenols bound to cereal dietary fiber. Am. J. Clin. Nutr. 101, 1-12.

Vitali, V., Ndagijimana, M., Cruciani, F., Carnevali, P., Candela, M., Guerzoni, M.E., Brigidi, P., 2010. Impact of a synbiotic food on the gut microbial ecology and metabolic profiles. BMC Microbiol. 10, 4.

Vivas, S., Ruiz de Morales, J.M., Fernandez, M., Hernando, M., Herrero, B., Casqueiro, J. Gutierrez, S., 2008. Age-related clinical, serological, and histopathological features of celiac disease. Am. J. Gastroenterol. 103, 2360-2365.

Voreades, N., Kozil, A., Weir, T.L., 2014. Diet and the development of the human intestinal microbiome. Front. Microbiol. 22 (5), 494.

Wacklin, P., Laurikka, P., Lindfors, K., Collin, P., Salmi, T., Lähdeaho, M.L., Saavalainen, P., Mäki, M., Mättö, J., Kurppa, K., Kaukinen, K., 2014. Altered duodenal microbiota composition in celiac disease patients suffering from persistent symptoms on a long-term gluten-free diet. Am. J. Gastroenterol. 109, 1933-1941.

Want, E.J., Nordstrom, A., Morita, H., Siuzdak, G., 2007. From exogenous to endogenous: the inevitable imprint of mass spectrometry in metabolomics. J. Proteome Res. 6, 459-468.

Winder, C.L., Cornmell, R., Schuler, S., Jarvis, R.M., Stephens, G.M., Goodacre, R., 2011. Metabolic fingerprinting as a tool to monitor whole-cell biotransformations. Anal. Bioanal. Chem. 399, 387-401.

Zamakhchari, M., Wei, G., Dewhirst, F., Lee, J., Schuppan, D., Oppenheim, F.G. Helmerhorst, E.J., 2011. Identification of Rothia bacteria as gluten-degrading natura colonizers of the upper gastro-intestinal tract. PLoS One 6, e24455.

Zarco, M.F., Vess, T.J., Ginsburg, G.S., 2012. The oral microbiome in health and disease and the potential impact on personalized dental medicine. Oral Dis. 8, 109-120.

Zhang, A., Sun, H., Wang, X., 2012. Saliva metabolomics opens door to biomarker discovery, disease diagnosis, and treatment. Appl. Biochem. Biotechnol. 168, 1718-1727.

Zoetendal, E.G., Raes, J., van den Bogert, B., Arumugam, M., Booijink, C.C., Troost, F.J., Bork, P., Wels, M., de Vos, W.M., Kleerebezem, M., 2012. The human small intestinal microbiota is driven by rapid uptake and conversion of simple carbohydrates. ISME J. 6 , 1415-1426. 